\title{
Cartão Nacional de Saúde - os desafios da concepção e implantação de um sistema nacional de captura de informações de atendimento em saúde
}

\author{
National Health Card - the implementation \\ and conception challenges of a national system \\ used to extract information from health services
}

Rosani Evangelista da Cunha 1

1 Secretaria de Gestão de Investimentos do Ministério da Saúde. Esplanada dos Ministérios, Bloco G, sala 102, 70058-900 Brasília DF. rosani.evangelista@saude. gov.br
Abstract The National Health Card, currently implemented as a pilot project collects health encounter data at the point of care, allowing for the construction of the national repository of clinical data and the retrieval of information and knowledge to support health care policy makers in their daily decisions. The development of the system, and the strategies used in its implementation are based in principles that lead to specific technological options; among these, the following should be mentioned: a) the possession of the card is not a condition to access the health services; $b$ ) the access to information respects the user's privacy and confidentiality and the code ethics of each health professional category; (c) SUS staff is responsible for safeguarding the database; (d) adoption of open standards and; (e) respect to the work flow and functionality of the health care units. About 13 million people are directly affected by the pilot project that started in 1999 through an international bidding for the National Health Card informatics solution. These paper describes the pilot project from 1999 to 2003, date scheduled for completing the pilot deployment in 44 municipalities of the pilot project. The challenges and the project management complexity are discussed in this paper.

Key words Information system, Health information, Health informatics, Public informatics, Interoperability, Health informatics standards, XML.
Resumo O Cartão Nacional de Saúde, em implantação sob a forma de projeto piloto, utiliza tecnologias de informática para a captura de informações de atendimento em saúde. $O$ desenvolvimento do sistema e as estratégias utilizadas para sua implantação têm sido pautados por princípios que levaram a opções tecnológicas específicas. Merecem destaque: a) o porte do cartão não é condição para acesso aos serviços de saúde; $b$ ) o acesso às informações deve respeitar o direito à privacidade dos usuários e a ética dos profissionais; c) a responsabilidade pela guarda da base de dados é dos gestores do SUS; d) adoção de padrões, preferencialmente abertos; e e) respeito ao processo de trabalho e às funcionalidades requeridas no ato do atendimento. Cerca de 13 milhões de pessoas são abrangidas de forma direta pelo projeto piloto, que teve origem em meados de 1999 por meio de uma licitação internacional para contratação da Solução de Informática do Cartão Nacional de Saúde. Com pouco mais de três anos de duração e previsão de término do projeto piloto para julho de 2003, o Cartão apresenta características e desafios próprios de projetos complexos, que são discutidos no presente artigo.

Palavras-chave Sistema de informação, Informação em saúde, Informática em saúde, Informática pública, Interoperabilidade, Padrões em saúde, XML 


\section{Contextualização}

A implantação do Cartão Nacional de Saúde é uma demanda histórica do setor saúde no Brasil. Previsto em instrumentos normativos do sistema de saúde brasileiro desde o ano de 1996, o objetivo do Cartão é a identificação e acompanhamento do conjunto de atendimentos realizados pelo sistema de saúde, independentemente dos locais de realização dos mesmos. Apesar de previsto desde 1996, a complexidade e o ineditismo do projeto fez com que somente em 1999 ele fosse iniciado, ainda sob a forma de Projeto Piloto.

O projeto, de grande complexidade e com cerca de três anos de duração, está em processo de desenvolvimento e implantação e não será finalizado no curto prazo. No entanto, a experiência em curso já proporcionou uma significativa experiência cognitiva, principalmente em virtude do aprendizado decorrente da articulação entre concepções teóricas e o processo prático de desenvolvimento e implantação de uma solução de abrangência nacional, com presença de diferentes componentes próprios de um projeto complexo de uso de tecnologias de informática e comunicação voltadas ao setor saúde.

\section{Princípios e diretrizes do Cartão Nacional de Saúde}

O Projeto Cartão Nacional de Saúde, cuja concepção, desenvolvimento e implantação são orientados pelo arcabouço que conforma o SUS, tem sido entendido como um instrumento fundamental para articular a execução descentralizada dos serviços e o caráter nacional e único do sistema de saúde. Não é por outro motivo que a primeira menção ao Cartão se deu na Norma Operacional Básica do SUS de 1996, instrumento que redesenhou as atribuições dos gestores, aprofundou o processo de descentralização e apontou para a necessidade de mecanismos de integração entre esferas de governo. A contribuição do Cartão na integração entre o local e o nacional é dada pela captura de informações no ato do atendimento prestado ao usuário e o acompanhamento do seu fluxo subseqüente, em cada contato deste usuário com o SUS, em qualquer localidade do país.

Os princípios de universalidade de acesso, integralidade de atendimento, eqüidade, democratização e descentralização do SUS, bem co- mo o direito do cidadão à preservação de sua autonomia, integridade moral e privacidade quanto às informações relacionadas à sua saúde, são as bases que norteiam a construção do Cartão Nacional de Saúde. Desses princípios derivam diretrizes do Sistema Cartão, que são traduzidas em opções tecnológicas e mecanismos de gestão e implantação específicos.

A primeira diretriz considerada, pautada pelo princípio da universalidade do direito à saúde, é que o porte do cartão não pode ser entendido como condição para acesso dos usuários aos serviços. A segunda diretriz é que o acesso às informações do sistema deve respeitar os aspectos de ética profissional e o direito à privacidade, confidencialidade e intimidade dos cidadãos. Uma terceira diretriz diz respeito ao uso de padrões abertos, de forma a permitir a integração do Cartão com iniciativas e sistemas já existentes e em desenvolvimento.

A preocupação com a interoperabilidade de sistemas de informação é um dos focos do projeto. A magnitude do sistema de saúde brasileiro, a diversidade de ações desenvolvidas e a demanda por informações específicas, a histórica dicotomia entre ações assistenciais e de promoção, o modelo de financiamento pautado pelo pagamento de procedimentos, dentre outros, fizeram com que o país contasse com dezenas de sistemas de informação de base nacional. Alguns desses foram construídos há décadas, na sua maioria voltados para o faturamento, com tecnologias antigas e sem qualquer preocupação com interoperabilidade. Ao longo do tempo, cada sistema impôs o seu conjunto de padrões para representar a informação, o que gera repetição do trabalho de coleta, visões fragmentadas e bases de dados pouco confiáveis. Este seguramente é um desafio a ser enfrentado pela área de informação e a superação dessa fragmentação tem sido uma preocupação no desenvolvimento do Cartão Nacional de Saúde.

Na perspectiva do governo federal, o Cartão Nacional de Saúde foi concebido como um sistema de informação que utiliza a informática e as telecomunicações com o propósito de identificar o usuário do SUS, integrar informações e construir a base de dados de atendimentos em saúde. Na perspectiva dos gestores estaduais e municipais, o Cartão pode apoiar a organização dos serviços em sua esfera de competência e facilitar a negociação e a gestão intergovernamental. O sistema pode possibilitar aos gestores do SUS, em qualquer espaço de inserção, o acesso e a geração de informações in- 
dividualizadas, referenciadas a diferentes bases territoriais e acompanhadas e controladas ao longo do tempo.

Um projeto dessa magnitude não pode ser construído sem uma perspectiva de longo prazo. Assim, ele necessita ser implementado em sucessivos ciclos, considerando fases de revisão, planejamento, implantação e análise de resultados. Tendo em vista o conceito de ciclos e a magnitude dos objetivos e do próprio sistema de saúde, o Ministério da Saúde optou por desenvolver um projeto piloto. O objetivo do $\mathrm{Pi}$ loto é aprofundar as definições conceituais do Sistema Cartão, desenvolver seus vários componentes e construir metodologia de implantação, de forma a permitir sua apropriação em escala nacional. Para tanto, foi realizada uma licitação para contratação da "Solução de Informática do Cartão Nacional de Saúde" e selecionados 44 municípios onde o sistema seria testado. Estes, localizados em 11 estados, abrangem cerca de 13 milhões de habitantes. A escolha privilegiou desde pequenas cidades a grandes centros urbanos, com serviços de alta complexidade.

A licitação previa a contratação de uma solução de informática global, dividida em três lotes, incluindo o desenvolvimento e instalação de terminais específicos, aquisição da infra-estrutura de informática e de comunicação, desenvolvimento de aplicativos, emissão dos cartões, capacitação de recursos humanos, manutenção e assistência técnica, dentre outros.

\section{O processo de desenvolvimento do sistema}

Apesar de se caracterizar como um sistema de informação de base nacional, o Cartão Nacional de Saúde captura a informação no ato do atendimento ao usuário. Ou seja, o dado é produzido de forma descentralizada, no contato entre o profissional e o usuário atendido, no cotidiano dos serviços. O objetivo do projeto é a identificação do atendimento realizado, vinculando-o ao usuário, ao profissional que o atendeu e à unidade de saúde. Assim, e como forma de garantir confiabilidade para os dados e para as informações produzidas, as principais decisões tomadas ao longo do desenvolvimento foram relacionadas às seguintes indagações: (a) como identificar de maneira unívoca os usuários? (b) quais dados devem ser capturados para caracterizar um atendimento e quais funcionalidades devem ser desenvolvidas? (c) quais padrões devem ser adotados para representar e transmitir a informação, tornando os dados capturados comparáveis e garantindo interoperabilidade com outros sistemas?

\section{A identificação dos usuários}

Para a identificação dos usuários do SUS está sendo constituída uma base de dados cadastrais e, a partir daí, são gerados números de identificação para cada cidadão. Considerando que a população busca serviços em diferentes municípios, é de fundamental importância que a identificação dos usuários seja vinculada a um município, mas tenha validade nacional. É este o objetivo do cadastramento, gerar um número nacional de identificação, mas vinculado ao município de residência do cidadão. Este número é impresso no cartão do usuário e permite sua identificação sempre que buscar serviços no SUS.

O Cartão SUS também identifica os profissionais de saúde e as unidades onde os atendimentos são realizados. Para tanto, os profissionais também são cadastrados e recebem seus cartões de identificação, com a diferença de que possuem senha individual, sigilosa e intransferível. Esta senha, a partir da qual é permitido o acesso ao sistema para registro e consulta aos dados, é gravada de forma criptografada na tarja magnética do cartão e permite a identificação do profissional que faz atendimento, lança ou extrai dados do sistema. Assim, os registros coletados pelo sistema são vinculados a um determinado profissional. As unidades são identificadas por meio do Cadastro Nacional de Estabelecimentos Assistenciais de Saúde.

\section{Os dados capturados \\ e as funcionalidades desenvolvidas}

Diferentemente dos sistemas de informação tradicionais, o Cartão SUS não captura dados visando ao faturamento ou exclusivamente à vigilância epidemiológica. O Cartão trabalha com o conceito de captura de informações de atendimento, entendido como o resultado do encontro entre um usuário e um profissional, vinculado a um determinado estabelecimento de saúde, que, por sua vez, vincula-se a um determinado território. Compõem ainda este atendimento as informações referentes ao problema identificado, o procedimento realizado, o medicamento prescrito e dispensado e o que resultou do atendimento. 
Com o objetivo de adequar o sistema às situações cotidianas dos profissionais e das unidades, identificando as funcionalidades e os dados a serem capturados para representar o atendimento, foi utilizada a metodologia de descrição de casos de uso, a UML (Unified Modeling Language). Como resultado do trabalho foram identificados cenários específicos para o atendimento nos serviços de atenção básica, de média e alta complexidade e nos serviços hospitalares. Assim, os dados capturados e as funcionalidades desenvolvidas são adequados ao processo de trabalho no estabelecimento específico. De forma geral, os dados capturados permitem formular respostas às seguintes perguntas:

- Quem foi atendido.

- Quem atendeu este usuário.

- Onde e quando o usuário foi atendido.

- Qual o problema de saúde identificado.

- Qual o procedimento realizado.

- Qual medicamento foi prescrito e qual foi dispensado.

- Os motivos da procura e o que resultou do atendimento.

Além da captura dessas informações, no nível de atendimento foram desenvolvidas as funcionalidades de cadastramento e identificação de usuários; habilitação e autenticação de profissional de saúde; localização de prontuário; agendamento local; autorização de procedimentos; registros de prescrição e dispensação de medicamentos, de diagnósticos, de procedimentos terapêuticos, de notificação de eventos e agravos; emissão de boletos para os usuários (como comprovante de solicitação de medicamentos, marcação de consulta, resumo de atendimento) e extratos referentes a atendimentos realizados para os profissionais (inclusive histórico clínico dos usuários).

No espaço da gestão, por meio dos dados armazenados nos equipamentos servidores, $\mathrm{o}$ sistema permite a geração de qualquer tipo de relatório ou informação resultantes do conjunto de dados capturados e do relacionamento entre eles. Podem ser relatórios (pré-formatados ou não), consultas dinâmicas à base de dados, acompanhamento de situações marcadoras e de eventos sentinelas, etc. Ainda nos servidores podem ser feitas configuração e auditoria do acesso às bases de dados, o cadastramento de profissionais e de estabelecimentos, além da exportação de dados para outros sistemas.

A transformação dos dados em informação depende da capacidade de formulação dos profissionais que têm acesso às bases de dados. $\mathrm{Na}$ perspectiva dos dirigentes, o Cartão disponibiliza informações que podem contribuir para uma melhor organização e racionalização de serviços; para a democratização da gestão; para o aperfeiçoamento dos mecanismos de controle, avaliação e auditoria; para a organização (reorganização) de referências e revisão de critérios de financiamento; permite acompanhar a demanda por serviços e por medicamentos, subsidiando decisões de compras e alocação de insumos. É possível acompanhar os problemas de saúde da população de forma dinâmica, longitudinal e vinculada a um território, com repercussões na escolha de ações que melhor respondam à resolução de tais problemas. Enfim, uma série de possibilidades se abre e essas possibilidades são dependentes da capacidade dos dirigentes em fazer perguntas e interpretar as informações disponibilizadas pelo sistema.

Do ponto de vista dos profissionais de saúde, o cartão pode apoiá-los no atendimento individual, ao disponibilizar o histórico clínico do usuário com informações pregressas sobre contatos com os serviços, diagnósticos anteriores, medicamentos recebidos e encaminhamentos para outros procedimentos. Estas informações podem facilitar diagnósticos e indicação de condutas clínicas. Pode, ainda, contribuir para uma racionalização do atendimento, ao mostrar, por exemplo, que determinado procedimento já foi realizado e não deve ser novamente solicitado. Ao longo do tempo, o acompanhamento da base pode apoiar a construção de protocolos clínicos, o que seria de grande valia para o setor saúde.

Na perspectiva dos usuários, o Cartão pode contribuir para uma melhor organização dos serviços segundo suas demandas, para uma maior agilidade no atendimento e na marcação de consultas e exames, para uma melhor interação com os profissionais que o atendem, que passam a ter acesso ao seu histórico clínico, dentre outros. Além disto, o usuário passa a ter em mãos um instrumento de materialização do seu direito à saúde.

É óbvio que estes ganhos não são automáticos e que o Cartão não é um instrumento que resolva de forma mágica os problemas do sistema de saúde. A experiência do projeto piloto, no entanto, mesmo incipiente, tem mostrado diferentes usos para o Cartão Nacional de Saúde, alguns até não imaginados anteriormente pelo Ministério da Saúde. 


\section{Os padrões adotados pelo sistema}

O terceiro grupo de decisões presente no desenvolvimento do sistema diz respeito aos padrões de representação do conjunto de atos que compõem o atendimento em saúde. Sem esta forma de representação não existiriam possibilidades de captura da informação ou elas não seriam comparáveis. Na definição dos padrões, o Cartão Nacional de Saúde considerou não apenas os aspectos de hardware e software, mas também a representação, transmissão, acesso e armazenamento da informação. Assim, o projeto trabalhou com padrões específicos da saúde, abrangendo vocabulários, estrutura e conteúdo, e aqueles relacionados aos aspectos computacionais, como hardware, arquitetura de rede, conectividade e segurança.

Para descrever a informação em saúde, condição para o registro dos atendimentos e para os cadastros, o sistema adotou padrões de vocabulário. Para cada informação capturada, o Cartão definiu tabelas específicas, que descrevem conceitos em saúde representados por um código e respectiva descrição. Nesse processo foram adotados padrões de vocabulário formalizados ou já consagrados pelo uso, mas houve também demanda de padrões para áreas onde os mesmos ainda não existiam e, ainda, necessidade de adequação de alguns padrões às especificidades de um formato de captura descentralizada, no ato do atendimento.

Tendo em vista que o Cartão definiu novas tabelas para registrar o atendimento, houve necessidade de estabelecer mapeamentos para tabelas utilizadas por outros sistemas, com vistas a garantir compatibilização entre eles. Ao mesmo tempo, e levando em conta o caráter descentralizado do SUS, o sistema também permite graus de flexibilidade, possibilitando a inclusão de itens de interesse local em espaços específicos das tabelas.

Com a implantação do Projeto Cartão Nacional de Saúde inicia-se a criação do repositório nacional de dados de atendimento, distribuído nas três esferas de governo, com servidores próprios para cada esfera. Este passa a ser o principal padrão de conteúdo da informação em saúde no país. Todas as aplicações que desejarem integrar-se ao Projeto Cartão deverão ser capazes de gerar este conjunto de informações.

Os padrões de comunicação, que definem o formato e conteúdo das mensagens, protocolo de transmissão e requisitos de telecomunicação, também são fundamentais no Cartão, per- mitindo as transações no interior do sistema e a interoperabilidade com outros sistemas de informação. O projeto adotou o XML - eXtended Mark-up Language como padrão para troca, publicação e armazenamento. O XML permite o processamento inteligente de documentos, a apresentação e pesquisa baseada em conteúdo, a independência de plataformas de hardware e software e o compartilhamento e reutilização da informação. A interoperabilidade entre sistemas é garantida a partir da definição de modelos comuns que definem a estrutura dos documentos XML, as DTD - Document Type Definition.

Em relação aos padrões de segurança, o projeto adotou padrões referentes à privacidade, confidencialidade, autenticidade, integridade dos dados de saúde e auditoria.

\section{Aspectos tecnológicos do projeto}

Do ponto de vista tecnológico, a Solução de Informática do Cartão Nacional de Saúde está baseada em cinco componentes principais: os cartões de identificação dos usuários e dos profissionais; a rede de comunicação; os terminais de atendimento e os equipamentos para armazenamento e gerenciamento da base de dados; os aplicativos do nível de atendimento e dos servidores e; os aspectos de segurança e a política de controle de acesso.

Os cartões de usuários e de profissionais de saúde são o elemento de identificação dos mesmos perante o sistema. São cartões de tarja magnética de alta coercitividade, contendo o número e o nome do cidadão, sexo, data de nascimento e município de residência. Os cartões são, portanto, instrumentos de identificação e não de armazenamento de informações. O cartão do profissional traz embutida uma senha criptografada que permite acesso ao sistema para registro e consulta de dados. Estes cartões são lidos por um terminal especialmente desenvolvido para o projeto, os Terminais de Atendimento do SUS - TAS.

Com o objetivo de refletir a organização do SUS, o Sistema Cartão Nacional de Saúde desenvolveu uma arquitetura que prevê a existência de bases de dados nos âmbitos federal, estadual e municipal. Em cada um desses níveis foram instalados servidores e demais equipamentos que permitem o armazenamento do conjunto dos dados dos atendimentos realizados. No nível de atendimento, ou seja, nos estabele- 
cimentos de saúde, são instalados os terminais para captura dos dados. Entre as esferas de governo foi instalada uma rede de comunicação com tecnologia frame relay, configurada como uma rede TCP/IP Classe A tipo Intranet, com endereçamento 10.X.X.X desde o nível federal até o nível municipal. Entre os terminais de atendimento e o servidor municipal as comunicações são feitas por linha discada, o mesmo acontecendo entre o servidor municipal e o servidor concentrador. Em decorrência da preocupação do projeto com os aspectos de segurança, toda a comunicação entre os equipamentos é cifrada e autenticada e a conexão entre roteadores da rede é realizada por meio de uma VPN, utilizando-se o protocolo IPSec (Internet Protocol Security). Além disso, o tráfego de dados entre servidores, assim como todo o tráfego de serviço (consultas) é realizado sobre o protocolo HTTPS, ou seja, protocolo HTTP (Hypertext Transfer Protocol) sobre o protocolo SSL (Secure Sockets Layer).

Do ponto de vista tecnológico, uma inovação do projeto está no desenvolvimento do Terminal de Atendimento da Saúde - TAS. Este funciona como entrada do sistema, para registro de dados e emissão de relatórios. A criação do TAS teve como objetivo garantir um terminal fácil de ser utilizado, adequado ao perfil de unidades que funcionam com infra-estrutura elétrica e de telefonia precária e, ainda, possibilitar maior segurança em relação ao acesso. $\mathrm{O}$ desenvolvimento do TAS considerou requisitos de robustez, interface amigável, armazenamento em memória não volátil, segurança, opções de funcionamento on-line e off-line, interfaces para impressoras e outros periféricos, adesão a padrões abertos, dentre outros.

No caso de municípios que dispõem de sistema de informação local que captura os dados do Cartão, normalmente com uso de microcomputadores, é possível a integração entre os sistemas. Assim, apesar de ter desenvolvido um equipamento específico, o sistema Cartão não é dependente do TAS. O Ministério da Saúde não impõe a utilização do equipamento e mesmo do aplicativo, mas estabelece padrões de interoperabilidade, através da troca de documentos XML (eXtended Mark-up Language).

\section{Aquisição e desenvolvimento dos aplicativos}

Desenvolver software é uma tarefa árdua, especialmente para domínios de alta complexi- dade. A área da saúde, diferentemente de áreas onde as informações são bem definidas e os dados são simples, impõe enormes desafios à equipe de desenvolvimento. Em primeiro lugar está o desafio de representar a informação do ato assistencial. Faltam vocabulários adequados para descrever a complexidade semântica da área. Além dos vocabulários, a definição do conteúdo de dados a ser capturado também não existia quando da licitação. No início do desenvolvimento, alguns requisitos ficaram claros: o sistema deveria ser flexível, aberto, utilizar XML para a troca de informação e, acima de tudo, seguro.

Os aplicativos desenvolvidos e adquiridos para o projeto compreendem o software do TAS, os aplicativos de gestão municipal, estadual e federal e os gerenciadores de rede. Para extrair a informação dos bancos de dados foi adquirida uma ferramenta Information System (EIS), destinada a facilitar a geração de relatórios, segundo a demanda de cada gestor. Para garantir a independência quanto às tecnologias e soluções proprietárias, a comunicação entre os terminais e os servidores é implementada obedecendo a padrões abertos definidos por organismos como o IETF ou o Consórcio W3 (TCP/IP, PPP, HTTPS, SSL, XML).

Do ponto de vista da arquitetura de software que garante a infra-estrutura, a opção foi utilizar uma arquitetura multicamada, baseada em interfaces padronizadas. Muito mais do que uma solução específica de software, o sistema Cartão Nacional de Saúde é, na realidade, um framework para a saúde, a partir do qual outros sistemas podem ser construídos. Este framework, com uma clara distinção entre as camadas da arquitetura (apresentação, negócios e persistência), permite expandir, criar novas funcionalidades e reutilizar o código.

A adoção de arquitetura de software robusta e a metodologia de desenvolvimento que garantisse escalabilidade e flexibilidade do produto final foram fatores decisivos para o desenvolvimento. Afinal, trata-se de projeto de longo prazo, em área complexa, com abrangência nacional, ainda que sob forma de piloto. Toda a aplicação do Sistema Cartão é de propriedade do Ministério da Saúde, com código fonte aberto. Sem dúvida, a forma de desenvolvimento adotada pelo Cartão concretiza as definições propostas como base do Sistema Nacional de Informação em Saúde - SNIS. 


\section{Política de acesso às informações do cartão}

Para a definição da Política de Acesso às Informações do Cartão Nacional de Saúde, o Ministério da Saúde se deparou com preocupações e com escolhas à primeira vista contraditórias. Era necessário fazer escolhas ou buscar equilíbrio entre, por exemplo, direitos individuais e necessidades coletivas, entre necessidade de acesso e garantia de confidencialidade e privacidade, entre demandas decorrentes da assistência e aquelas ligadas à gestão e à regulação do sistema de saúde. De um lado, existem os direitos consagrados da sociedade brasileira ao conhecimento, à transparência e à democratização da informação componente da gestão do SUS. Por outro, está o direito do cidadão ao sigilo, à intimidade e à confidencialidade em relação às informações de saúde identificadas.

Os avanços e potencialidades da tecnologia não são suficientes para responder a estes conflitos. Esta também não pode ser uma decisão de setores isolados. Numa sociedade democrática, a definição sobre Política de Acesso deve se dar a partir da mais ampla discussão em torno do assunto, preferencialmente pelo Congresso Nacional. Em geral, esta é uma discussão de longo prazo, conforme demonstra a experiência internacional. Enquanto o país não dispõe de legislação específica sobre o assunto, o Ministério da Saúde elaborou uma proposta preliminar de Política de Acesso e a submeteu à consideração de diferentes segmentos ligados ao setor saúde e àqueles que atuam na garantia de direitos de cidadania.

A elaboração da Política de Acesso foi precedida de análise da legislação sobre direito à intimidade, privacidade, ética e sigilo, considerando de forma diferenciada a captura, acesso e divulgação de informações. Buscou subsídios também na regulamentação existente no país sobre atuação profissional, em especial aquela referente aos códigos de ética dos profissionais do setor saúde. À tecnologia coube implementar os perfis e as salvaguardas definidas pelos profissionais, dirigentes, juristas e demais interlocutores nesta discussão.

Como princípio, foi considerado que os dados e informações clínicas individuais pertencem ao cidadão ao qual se referem. Devem ser garantidos a essa pessoa a privacidade e o sigilo profissional em relação aos seus dados e informações, por parte de todos os profissionais de saúde direta e indiretamente envolvidos na atenção à sua saúde. Devem ser garantidos a confidencialidade, a integralidade e a segurança no registro, transmissão, armazenamento e utilização dos dados individuais existentes no serviço de saúde. A partir destas referências, a Política de Acesso às Informações do Cartão Nacional de Saúde considerou as seguintes diretrizes para regular o acesso aos dados, informações, cadastros, arquivos e bases de dados do Sistema, dentre outros:

a) Implementação de mecanismos que permitam o acesso a uma determinada informação somente às pessoas autorizadas a acessarem aquela informação;

b) Autorização de acesso às informações apenas para as pessoas que necessitarem daquela informação para exercer sua função ou executar seu trabalho;

c) Acesso permitido apenas em razão de necessidade da população ou do paciente, de necessidade do profissional ou de necessidade do administrador, nessa ordem e, ainda, por determinação judicial e no limite da lei;

d) Implementação de mecanismos de segurança, controle e auditoria, de forma a permitir o registro e o conhecimento sobre quem teve acesso, quando e a qual informação;

e) Permissão para o intercâmbio de dados, limitado às informações cadastrais, com outras instituições governamentais, desde que estas garantam o sigilo, a confidencialidade, a integridade e a segurança em seu tratamento e utilização;

f) Vedação de intercâmbio de dados, informações, cadastros e arquivos com operadoras de seguros, planos de saúde e outras instituições com fins comerciais;

g) Permissão de acesso aos dados, informações, cadastros e arquivos para instituições de pesquisa apenas em formato consolidado e não identificado.

Com base em tais diretrizes, e considerando as definições contidas em códigos de ética e conduta profissional, o acesso aos dados, cadastros e informações foi formatado segundo demandas específicas de categorias profissionais, o tipo de evento, o local de atendimento e o nível hierárquico. $\mathrm{O}$ acesso é dado, portanto, a partir de uma combinação entre os mecanismos de controle implementados no sistema e a caracterização de cada profissional, contida no seu cadastro. O reconhecimento dos profissionais é feito por meio do seu cartão e da senha.

Do ponto de vista tecnológico foram estabelecidos mecanismos com vistas à preserva- 
ção do sigilo, da privacidade, da confidencialidade, da autenticidade e da integridade dos dados de saúde. A tecnologia adotada possibilita, dentre outros, a incorporação das regras de acesso à informação em todos os níveis da rede e mecanismos que permitem a auditagem da veracidade e fonte das informações. Para que os requisitos de segurança sejam atendidos, a comunicação entre equipamentos (terminais ou servidores) é cifrada e autenticada.

Como parte da Política de Acesso às Informações do Cartão Nacional de Saúde, o Ministério da Saúde formatou um Termo de Compromisso e Responsabilidade, assinado pelos dirigentes da saúde e pelas empresas contratadas para execução de serviços no Projeto que, por algum motivo, possam ter acesso às bases de dados. Este documento formaliza a responsabilidade dos gestores com a guarda da base de dados e o compromisso com a não divulgação de cadastros e informações de atendimento individualizadas.

O documento de Política de Acesso às Informações do Sistema Cartão Nacional de Saúde representou um marco no país, uma vez que foi a primeira iniciativa de discussão ampliada e de revisão da legislação brasileira sobre os aspectos de sigilo, privacidade e confidencialidade da informação em saúde. A partir desta revisão, espera-se estimular uma discussão mais ampliada com vistas à definição de uma legislação específica sobre o assunto.

\section{Os principais desafios \\ no desenvolvimento e implantação do projeto}

Vários têm sido os desafios enfrentados na implantação do projeto, decorrentes do seu próprio grau de inovação, magnitude e complexidade. Parte expressiva desses desafios extrapola o setor saúde e demanda intervenção de Governo no sentido mais ampliado. A inexistência ou insuficiência de recursos humanos com capacitação na área de informática, a dificuldade de acesso e a baixa qualidade das linhas telefônicas, as definições quanto a padrões de informática e política de acesso, dentre outros, são exemplos de decisões/problemas que demandam atuação de outros segmentos e não apenas do setor saúde.

Apesar das dificuldades encontradas, a forma de enfrentamento dos problemas e a busca de alternativas coerentes com os princípios e diretrizes do SUS fizeram com que o projeto, após cerca de três anos de implantação, fosse reconhecido como bem-sucedido por diferentes interlocutores. A análise dos desafios encontrados, em especial num momento de transição de governo, pode contribuir para as definições futuras quanto à consolidação/expansão do Cartão Nacional de Saúde. Como forma de facilitar sua sistematização, são considerados quatro grupos distintos de desafios: os conceituais, operacionais, de gestão, e aqueles relacionados à garantia de sustentabilidade do projeto.

\section{Os desafios conceituais}

O sistema de saúde brasileiro tem larga experiência com a captura e o uso de informações. Esta experiência, no entanto, está muito voltada para o acompanhamento da produção de serviços com vistas ao faturamento. O principal conceito utilizado pelo Cartão Nacional de Saúde, de captura dos dados de atendimentos realizados no âmbito do SUS, difere de forma significativa da experiência anterior do sistema de saúde.

O desenvolvimento do sistema Cartão a partir desse conceito não foi tarefa simples. Identificar o conjunto de informações que caracteriza o atendimento em saúde, definir padrões para representar essas informações, desenvolver funcionalidades de forma a refletir o processo de trabalho nas unidades de saúde e facilitar o uso, dentre outros requisitos, demandou significativo esforço de elaboração e de negociação por parte da equipe de condução do projeto. Agregado a isto, o baixo grau de definição do conteúdo e das intervenções em saúde tornou a tarefa mais complexa que em outras áreas de governo. A necessidade de refletir demandas assistenciais e administrativas, diferenciadas segundo a esfera de governo, gerou conflitos na definição do conjunto mínimo de dados que deveriam ser capturados e dificultou a construção de consensos em relação às prioridades do sistema.

Os conceitos trabalhados ao longo do projeto e a forma como se deram as negociações e a tomada de decisões, no entanto, fizeram com que as funcionalidades definidas para o projeto e os padrões adotados servissem de referência para a reformulação de diferentes sistemas de informação utilizados no âmbito do SUS.

Também pode ser considerado um desafio conceitual a decisão, ainda que provisória, quanto às formas de acesso às informações do Cartão Nacional de Saúde. 


\section{Os desafios operacionais}

Dentre os desafios operacionais podem ser identificados processos internos à própria equipe de condução do projeto no Ministério da Saúde. O cronograma de desenvolvimento e de implantação adotado, incompatível com a complexidade do projeto, e a demanda por trabalho intimamente integrado entre as áreas de saúde e informática poderiam ser definidos como duas das dificuldades mais expressivas internas à coordenação do projeto.

Em relação ao processo de implantação de forma mais específica, podem ser citadas as dificuldades decorrentes da demanda por sincronicidade entre diferentes frentes de trabalho para a implantação (cadastro, cartões, senhas, treinamento, instalação de equipamentos, etc.); a precariedade das unidades de saúde onde o sistema está sendo implantado, em especial no que se refere à infra-estrutura (acesso a linhas telefônicas de qualidade, por exemplo); a explicitação de processos não organizados ou fora dos parâmetros esperados nas unidades de saúde; a apresentação, por parte dos profissionais e dos dirigentes de saúde, de demandas para a organização e/ou reorganização dos serviços de saúde que não seriam de responsabilidade do projeto.

Os problemas relacionados aos recursos humanos e às dificuldades de cadastramento são, no entanto, os mais expressivos. Em relação aos recursos humanos, o projeto deparouse tanto com a inexistência ou insuficiência de recursos humanos, quanto com a inadequação de perfil. As atividades de capacitação desenvolvidas não são suficientes para resolver o problema e, num grande número de vezes, os profissionais qualificados pelo projeto, ao ganharem maior capacidade de competição no mercado, se desligam dos serviços de saúde. Em relação ao cadastramento, a complexidade do processo de identificação dos cidadãos foi superior ao esperado. Os problemas vão desde a existência de um grande número de pessoas sem documento de identificação, condição para o cadastramento, até as dificuldades para aperfeiçoar mecanismos de crítica que garantissem a confiabilidade da base de dados. Esta atividade, sem sombra de dúvidas, é das mais complexas e importantes do projeto.

As resistências corporativas, principalmente aquelas relacionadas à rede de prestadores privados de serviços de saúde e à corporação médica, estão sendo menos expressivas que o esperado pelo Ministério da Saúde.

\section{Os desafios de gestão}

Os desafios da gestão do projeto são principalmente de duas ordens. Aqueles decorrentes da necessidade de negociação interna (com setores do Ministério da Saúde) e externa (estados, municípios, entidades de profissionais e outros segmentos, dentre outras), e aqueles relacionados ao gerenciamento dos contratos junto aos fornecedores.

A necessidade de constituição de mecanismos permanentes de negociação e de construção de consensos é intrínseca a projetos da envergadura e da natureza do Cartão Nacional de Saúde. Várias são as decisões que demandam negociação mais ampliada, como adoção de padrões, política de acesso, definição de estratégias de implantação e responsabilidades de cada ente federado, interoperabilidade entre sistemas, dentre outras. Estas decisões demandam interlocução interna ao setor saúde e interlocução com áreas como ciência e tecnologia, comunicações, justiça, defesa da cidadania e outras.

Do ponto de vista da gestão dos contratos firmados com fornecedores, a principal preocupação foi construir salvaguardas que assegurassem que as decisões tomadas no desenvolvimento não criariam reservas de mercado no fornecimento de qualquer componente do sistema, capaz de restringir a concorrência em etapas posteriores. Ao mesmo tempo, foi necessário responder às demandas da legislação nacional e do organismo financiador no que se refere a recursos financeiros, processos de contratação, administração de patrimônio, importação, auditoria, dentre outros, além de garantir conformidade entre o que foi contratado e o que está sendo fornecido. A contratação de uma empresa de fiscalização externa, com funções de auditoria da solução de informática, foi de suma importância nesse processo.

\section{Os desafios para garantir sustentabilidade ao projeto}

A garantia de sustentabilidade do projeto no médio e longo prazo parece ser dependente de algumas condições, a primeira delas a própria finalização do processo de implantação do projeto piloto. Outras condições têm sido identificadas e trabalhadas pela coordenação do projeto na perspectiva de futuro, dentre elas o apoio aos estados e municípios participantes do projeto piloto no uso e gestão das informações derivadas do sistema; a incorporação do 
número do Cartão do usuário e dos profissionais nos sistemas de informação de base nacional; o apoio à integração de sistemas de informação; a avaliação do processo de implantação em curso e a identificação de alternativas que permitam, num segundo ciclo do projeto, uma maior adequação do sistema às condições encontradas em campo durante o piloto. O êxito dessas alternativas, no entanto, é dependente de mudanças na forma como o Ministério da Saúde, e o próprio SUS, conduz a área de informação em saúde. A unificação do comando da área de informação, articulando os conteúdos derivados da área de tecnologia e aqueles próprios do setor saúde, parece ser um importante passo na consolidação dessas mudanças.

A experiência observada em grandes projetos que utilizam tecnologia de informática e de telecomunicações mostra que o sucesso de tais iniciativas é dependente da consistência e da continuidade do suporte político. Isto é fundamental para a continuidade dos investimentos e para a capacidade de atualização, ampliação e manutenção. O Cartão Nacional de Saúde tem apoio explícito dos dirigentes do setor saúde de estados e municípios, possível de ser observado na adesão praticamente unânime ao processo de cadastramento de usuários em curso. No âmbito federal, além do respaldo dado pelos dirigentes do Ministério da Saúde, o apoio à implantação e expansão do Cartão Nacional de Saúde está presente nos programas de governo dos principais candidatos à Presidência da República. O projeto deixa de ser uma demanda específica do setor saúde, para ser uma iniciativa de governo. Assim, a garantia de continuidade do projeto não parece ameaçada, embora os cenários possíveis para sua consolidação e ampliação não estejam claramente definidos.

\section{Referências bibliográficas}

Brasil 1996. Gestão plena com responsabilidade pela saúde do cidadão (Norma Operacional Básica do SUS 01/96). Ministério da Saúde, Brasília.

Brasil 1999. Edital - Solução de Informática para Implementação do Cartão Nacional de Saúde (Republicação). Ministério da Saúde, Brasília.

Brasil 2001. Diretrizes para regulamentar o acesso aos dados e informações do Sistema Cartão Nacional de Saúde. Versão não publicada.

Dick RS, Steen EB, Detmer DE (eds.) 1997. The computer based patient record - an essential technology for health care. Committee on Improving the Patient Record. Institute of Medicine. National Academy Press, Washington DC.

Ferraz Júnior 1993. Sigilo de dados: o direito à privacidade e os limites da função fiscalizadora do Estado. Revista da Faculdade de Direito da Universidade de São Paulo, p. 440 ss.

Leão BF et al 2001. The Brazilian national health informatics strategy. In Proceedings. MEDINFO, Londres..

Leão BF 2000. Padrões para representar a informação em Saúde. Relatório Técnico. Projeto PNUD BRA/97/024 - SNIS - Sistema Nacional de Informação em Saúde.

Nardon FB 2000. Utilizando XML para a representação de informação em saúde. Newsletter SBIS.

Vanbemmel JH, Musen MA (eds.) 1997. Handbook of Medical Informatics. Springer-Verlag, Heidelberg, Alemanha. 